\title{
On the Equivalence of Orientation Error and Positive Definiteness of Matrices
}

\author{
Pål Johan From and Jan Tommy Gravdahl \\ Department of Engineering Cybernetics \\ Norwegian University of Science and Technology \\ 7491 Trondheim, Norway \\ from@itk.ntnu.no and tommy.gravdahl@itk.ntnu.no
}

\begin{abstract}
In this paper we show how a continuous set of orientations can be represented as a positive definiteness test on a given matrix. When this continuous set is restricted by the maximum allowed orientation error in some or all directions it is shown that the requirement for an orientation to satisfy these restrictions is equivalent to positive definiteness for a certain matrix. The problem of finding the optimal orientation that satisfies these restrictions is hence transformed into an optimisation problem on the Riemannian manifold of linearly constrained symmetric positive definite matrices. Thus, the problem of finding the optimal orientation can be solved as a standard optimisation problem with the constraints written in the form of linear matrix inequalities or barrier functions. Linear matrix inequalities have been extensively studied in the optimisation communities and good and efficient algorithms are available.
\end{abstract}

Index Terms-Robotics, Convex Optimization, LMI

\section{INTRODUCTION}

In a wide range of applications the orientation of a rigid body does not need to be restricted to one frame but can be given as a continuous set of frames. The attitude of a satellite can for example be set so that the transmitter or receiver points approximately in the direction of the earth. Another example is the end effector of a robotic manipulator where an orientation error is allowed in the end-effector orientation. In [1] the idea of introducing the paint quality as a constraint and minimise some additional cost function was presented. This opens for the possibility of allowing an orientation error in the orientation of the end effector in order to improve the speed of the job, reduce torques and so on. It was shown in [2] that by allowing an orientation error in the end effector configuration of a robotic manipulator, the speed and the quality of the job was improved. The orientation error was chosen intuitively, and the approach presented was not suitable for implementation in an optimisation algorithm. The pointing task is another example where a continuous set of orientations is allowed in the specifications of the end-effector orientation.

In [3] the problem of friction force limit constraints is transformed into a problem of testing for positive definiteness of a certain matrix. In this paper the same ideas are used to convert the problem of orientation error constraints into a test of positive definiteness of a matrix. For different types of orientation errors, a suitable matrix is found and it is shown that positive definiteness of this matrix is equivalent to an orientation that satisfies the given restrictions on the orientation.
By transforming the nonlinear orientation constraints into positive definiteness constraints imposed on certain symmetric matrices the problem of finding the optimal orientation is transformed into an optimisation problem on the smooth manifold of linearly constrained positive definite matrices. For the special case of positive definite symmetric matrices, the problem can be transformed into solving a linear matrix inequality (LMI). Convex optimisation problems involving LMIs have been extensively studied in literature, and good solutions, such as interior point algorithms, are known.

For many sets of orientations a symmetric matrix can be found very easily. For other sets with a more complicated structure, a symmetric matrix may be hard to find. It is a clear advantage to choose the sets for which a symmetric matrix can be found because LMIs of symmetric matrices are in general solved very efficiently. The difference between the sets for which a symmetric matrix can and cannot be found is shown through the examples.

The applications range from satellites and aircraft to robotics and rigid bodies in general. A satellite acted upon by an external force can be written as an LMI with an additional linear constraint. The cost function will typically tend to infinity at the border of positive definiteness, which is equivalent to orientation error constraints. In robotics, the freedom represented by the orientation can be used to improve the performance as in [2]. The advantage of the approach presented here is that the problem can be formulated as an optimisation problem on a smooth manifold. Hence, an optimal solution can be found, as opposed to the intuitively found geometric solution presented in [2].

The problems considered are formulated as maxdetproblems subject to LMI constraints. This is convenient when it comes to introducing several constraints on the orientation. A simple example when the directions of the $x$-axis and $z$-axis of the end-effector frame are specified independently is shown.

\section{REPRESENTING ROTATIONS}

Most of the fundamental principles of rotation were presented in two papers by Leonhard Euler in 1775 [4]. The first paper shows that any rotation can be accomplished by a sequence of three rotations about the coordinate axes. In the second paper, Euler states that any orientation can be represented by a rotation of some angle $\phi$ about some fixed 
axis $\boldsymbol{n}$. He also shows that the composition of two rotations is itself a rotation.

\section{A. The Unit Quaternion}

The unit quaternion representation closely relates to the results presented in Euler's second paper. A good introduction to quaternions is found in [5]. Any positive rotation $\phi$ about a fixed unit vector $\boldsymbol{n}$ can be represented by the four-tuple

$$
Q=\left[\begin{array}{c}
q_{0} \\
\boldsymbol{q}
\end{array}\right]
$$

where $q_{0} \in \mathbb{R}$ is known as the scalar part and $\boldsymbol{q} \in \mathbb{R}^{3}$ as the vector part. $Q(\phi, \boldsymbol{n})$ is written in terms of $\phi$ and $\boldsymbol{n}$ by

$$
q_{0}=\cos \left(\frac{\phi}{2}\right), \quad \boldsymbol{q}=\sin \left(\frac{\phi}{2}\right) \boldsymbol{n} .
$$

$Q$ is a quaternion of unit length and denoted a unit quaternion. Henceforth, all quaternions have unit length if not other is stated. Let $Q_{P}=\left[\begin{array}{ll}p_{0} & \boldsymbol{p}^{\top}\end{array}\right]^{\top}$. A multiplication of two quaternions is given by a quaternion product and is written in vector algebra notations as

$$
Q_{P} * Q=\left[\begin{array}{c}
p_{0} q_{0}-\boldsymbol{p} \cdot \boldsymbol{q} \\
p_{0} \boldsymbol{q}+q_{0} \boldsymbol{p}+\boldsymbol{p} \times \boldsymbol{q}
\end{array}\right] .
$$

The cross product implies that quaternion multiplication is not commutative, as expected. Let $Q_{P}=\left[\begin{array}{llll}p_{0} & p_{1} & p_{2} & p_{3}\end{array}\right]^{\top}$ and $Q=\left[\begin{array}{llll}q_{0} & q_{1} & q_{2} & q_{3}\end{array}\right]^{\top}$. Then the quaternion product is written as

$$
Q_{P} * Q=\left[\begin{array}{l}
p_{0} q_{0}-p_{1} q_{1}-p_{2} q_{2}-p_{3} q_{3} \\
p_{0} q_{1}+p_{1} q_{0}+p_{2} q_{3}-p_{3} q_{2} \\
p_{0} q_{2}+p_{2} q_{0}+p_{3} q_{1}-p_{1} q_{3} \\
p_{0} q_{3}+p_{3} q_{0}+p_{1} q_{2}-p_{2} q_{1}
\end{array}\right]
$$

The quaternion product of two unit quaternions is a unit quaternion. By the definition of the quaternion the quaternions $Q$ and $-Q$ produce the same rotation. This is referred to as the dual covering. The quaternion identity is given by $Q_{I}=\left[\begin{array}{llll}1 & 0 & 0 & 0\end{array}\right]^{\text {T. }}$.

A pure quaternion is a quaternion with zero scalar part. Any vector, $\overline{\boldsymbol{v}}=\left[\begin{array}{lll}x & y & z\end{array}\right]^{\top}$ can be represented by a pure quaternion

$$
\boldsymbol{v}=\left[\begin{array}{l}
0 \\
\bar{v}
\end{array}\right] \text {. }
$$

The conjugate of a quaternion is defined as

$$
Q^{*}=\left[\begin{array}{llll}
q_{0} & -q_{1} & -q_{2} & -q_{3}
\end{array}\right]^{\top} .
$$

\section{B. Quaternions and Rotations}

Let a vector, $\overline{\boldsymbol{v}}_{1}$, be represented by the pure quaternion $\boldsymbol{v}_{1}$. This vector can be rotated $\phi$ radians around the axis $\boldsymbol{n}$ by

$$
\boldsymbol{v}_{2}=Q * \boldsymbol{v}_{1} * Q^{*} .
$$

Every vector $\overline{\boldsymbol{v}} \in \mathbb{R}^{3}$ can be represented by a pure quaternion, hence $\boldsymbol{v}$ is not necessarily a unit quaternion. The quaternion, $Q(\phi, \boldsymbol{n})$, however, is unitary. This represents the angle and the axis that the vector $\overline{\boldsymbol{v}}_{1}$ is to be rotated about. The resulting vector, $\overline{\boldsymbol{v}}_{2}$, is then of the same length as $\overline{\boldsymbol{v}}_{1}$ if and only if $Q$ is a unit quaternion. The quaternion representation also leads to a useful formula for finding the shortest rotation from one orientation to another. Let $Q_{P}$ and $Q$ be two orientations. Then, by taking

$$
E=Q_{P}^{*} * Q,
$$

$E$ will rotate $Q_{P}$ into $Q$ by the shortest rotation.

Note that Equation (8) rotates one frame into another frame. By a frame it is meant a coordinate system in $\mathbb{R}^{3}$ using Cartesian coordinates. One frame with respect to another frame represents three degrees of freedom and is referred to as orientation. The inertial frame is denoted, $\mathcal{F}_{I}$ and the frame that correspond to the inertial frame by a rotation $Q$ from the inertial frame is denoted $\mathcal{F}_{Q}$. Equation (7) rotates one vector into another vector and has two degrees of freedom (e.g. longitude and latitude) [6]. A unit vector with respect to a unit reference vector is referred to as direction. Henceforth, the main concern is with the direction of the central axis, which is assumed to be the body frame $z$-axis of the end effector.

\section{Rotation Sequences}

In this paper, the orientation is represented by a rotation sequence of three rotations about the unitary axes. The ZYZsequence is given by first a rotation $\alpha$ about the $z$-axis followed by a rotation $\beta$ about the new $y$-axis. This describes the direction of the $z$-axis. The last degree of freedom is given by the rotation $\gamma$ about the $z$-axis. When the sequence is given, a one-to-one ${ }^{1}$ mapping between $(\alpha, \beta, \gamma)$ and the quaternion $Q=\left[\begin{array}{llll}q_{0} & q_{1} & q_{2} & q_{3}\end{array}\right]^{\top}$ can be found whenever $\beta \neq 0$.

Given a quaternion $Q$. Then $\alpha, \beta$ and $\gamma$ from the ZYZsequence are found by [8]

$$
\begin{gathered}
\alpha=\arctan 2\left(\frac{q_{2} q_{3}-q_{0} q_{1}}{q_{0} q_{2}+q_{1} q_{3}}\right), \\
\beta=2 \arcsin \sqrt{q_{1}^{2}+q_{2}^{2}}, \\
\gamma=\arctan 2\left(\frac{q_{2} q_{3}+q_{0} q_{1}}{q_{0} q_{2}-q_{1} q_{3}}\right) .
\end{gathered}
$$

The following relations are also used in the following:

$$
\begin{aligned}
& \alpha=\arctan \left(\frac{q_{3}}{q_{0}}\right)-\arctan \left(\frac{q_{1}}{q_{2}}\right), \\
& \gamma=\arctan \left(\frac{q_{3}}{q_{0}}\right)+\arctan \left(\frac{q_{1}}{q_{2}}\right),
\end{aligned}
$$

and hence

$$
\alpha+\gamma=2 \arctan \left(\frac{q_{3}}{q_{0}}\right) .
$$

The following lemmas will be used in the following to determine positive definiteness of a matrix.

Lemma 2.1: (Sylvester's criterion) A matrix $M$ is positive definite if and only if all of the leading principal minors are positive. $M$ is positive semi-definite if all the leading principal minors are non-negative.

\footnotetext{
${ }^{1}$ If the dual covering of the quaternion is taken into account, a one-to-two mapping can be found.
} 
Lemma 2.2: A block diagonal matrix

$$
P=\left[\begin{array}{cccc}
P_{1} & 0 & \ldots & 0 \\
0 & P_{2} & \ldots & 0 \\
\vdots & \vdots & \ddots & \vdots \\
0 & 0 & \ldots & P_{k}
\end{array}\right]
$$

is symmetric (semi) positive definite if and only if each block $P_{i}, \quad i=1, \ldots, k$ is symmetric (semi) positive definite.

\section{ORIENTATION ERROR CONSTRAINTS AS LMIS}

\section{A. Cone}

Assume that one would like to restrict the $z$-axis of $\mathcal{F}_{Q}$ to point in approximately the same direction as the $z$-axis of the inertial frame $\mathcal{F}_{I}$. This can be visualised by a cone of directions and restricted by $|\beta| \leq \beta_{\text {lim }}$ where $0 \leq \beta_{\text {lim }} \leq \pi$. The orientation error $\beta$ can be found only from $q_{1}$ and $q_{2}$ from the quaternion $Q$ by (10)

$$
\beta=2 \arcsin \sqrt{q_{1}^{2}+q_{2}^{2}} .
$$

Due to this observation, a test to verify if the $z$-axis of $\mathcal{F}_{Q}$ does not deviate from the $z$-axis of $\mathcal{F}_{I}$ by more than $\beta_{\text {lim }}$ is given in the following.

Proposition 3.1: Given a restriction in the orientation error, $\beta_{\text {lim }}$. Then the $z$-axis of $\mathcal{F}_{Q}$ rotated by $Q=$ $\left[\begin{array}{llll}q_{0} & q_{1} & q_{2} & q_{3}\end{array}\right]^{\top}$ from the inertial frame $\mathcal{F}_{I}$ lies within the restrictions given by $\beta_{\text {lim }}$ if and only if

$$
P=\left[\begin{array}{ccc}
\eta & 0 & q_{1} \\
0 & \eta & q_{2} \\
q_{1} & q_{2} & \eta
\end{array}\right] \succeq 0
$$

where $\eta=\sin \frac{\beta_{l i m}}{2}, 0 \leq \beta_{\text {lim }} \leq \pi$ and $\succeq$ means positive semi-definiteness of the symmetric matrix $P$.

Proof: As $\eta \geq 0$ and $\eta^{2} \geq 0$, from Lemma 2.1 it only remains to test for $\operatorname{det}(P)$. The determinant of $P$ is given by

$$
\operatorname{det}(P)=\eta\left(\eta^{2}-q_{1}^{2}-q_{2}^{2}\right) .
$$

Note that $0 \leq \beta_{\text {lim }}<\pi \Rightarrow \eta \geq 0$ so that $\operatorname{det}(P) \geq 0$ can be written as

$$
\begin{gathered}
\eta^{2}-q_{1}^{2}-q_{2}^{2} \geq 0 \\
\eta^{2} \geq q_{1}^{2}+q_{2}^{2} \\
\eta \geq \sqrt{q_{1}^{2}+q_{2}^{2}} \\
\sin \frac{\beta_{l i m}}{2} \geq \sqrt{q_{1}^{2}+q_{2}^{2}}
\end{gathered}
$$

As $0 \leq \sqrt{q_{1}^{2}+q_{2}^{2}} \leq 1 \Rightarrow 0 \leq \arcsin \sqrt{q_{1}^{2}+q_{2}^{2}}$, the following holds

$$
0 \leq 2 \arcsin \sqrt{q_{1}^{2}+q_{2}^{2}} \leq \beta_{\text {lim }}
$$

Then Equation (16) concludes the proof as

$$
0 \leq \beta \leq \beta_{\text {lim }}
$$

Similarly for strictly positive definiteness.
Note that the restrictions in Proposition 3.1 are on the directions of the $z$-axis only and that rotations about the $z$ axis itself are not restricted (the pointing task). Note also that $P$ is a symmetric matrix. This is an important property that will be used in the next sections.

\section{B. Restriction on the Orientation about the Central Axis}

In the following a condition on the orientation error about the central axis is given. Assume that the $x$-axis points in the direction of the velocity and that it is desired that the body frame $x$-axis points in approximately the direction of the $x$-axis of the reference orientation. Again consider the ZYZsequence. In the case when no orientation error is allowed for the direction of the central axis, this is given trivially by $|\gamma|<c_{\max }$, where $c_{\max }$ is the maximum allowed orientation error of the $x$-axis. For the ZYZ-sequence the direction of the $x$-axis is given by both $\alpha, \beta$ and $\gamma$. Assume that the orientation error of the direction of the $z$-axis is restricted as in the previous section. When this is restricted to be relatively small, the error in the direction of the $x$-axis can be approximated by the error in the orientation about the central axis. This error is written as

$$
\epsilon=\alpha+\gamma
$$

This leads to the following result.

Proposition 3.2: Assume that the orientation error of the direction of the $z$-axis is small. Given a restriction in the orientation error $\epsilon_{\text {lim }}$ about the central axis, the $x$-axis of $\mathcal{F}_{Q}$ rotated by $Q=\left[\begin{array}{llll}q_{0} & q_{1} & q_{2} & q_{3}\end{array}\right]^{\top}$ from the inertial frame $\mathcal{F}_{I}$ lies within the restrictions given by $\epsilon_{\text {lim }}$ if and only if

$$
P=\left[\begin{array}{cc}
\kappa & \frac{q_{3}}{q_{0}} \\
\frac{q_{3}}{q_{0}} & \kappa
\end{array}\right] \succeq 0
$$

where $\kappa=\tan \frac{\epsilon_{l i m}}{2}$.

Proof: The determinant of $P$ is given by

$$
\operatorname{det}(P)=\kappa^{2}-\frac{q_{3}^{2}}{q_{0}^{2}}
$$

As $0 \leq \tan \frac{\epsilon_{l i m}}{2}$ for $0 \leq \epsilon_{l i m} \leq \pi$, $\operatorname{det}(P) \geq 0$ is written as

$$
\begin{gathered}
\kappa^{2} \geq \frac{q_{3}^{2}}{q_{0}^{2}} \\
\kappa \geq\left|\frac{q_{3}}{q_{0}}\right| \\
\tan \frac{\epsilon_{l i m}}{2} \geq\left|\frac{q_{3}}{q_{0}}\right| \\
\epsilon_{\text {lim }} \geq\left|2 \arctan \left(\frac{q_{3}}{q_{0}}\right)\right|
\end{gathered}
$$

Then Equation (14) concludes that

$$
\epsilon_{l i m} \geq|\epsilon|
$$

where $\epsilon$ is given by Equation (14). 


\section{Direction of the $x$-axis}

Alternatively, one might want to restrict the direction of the $x$-axis directly. Note that the matrix given in the previous section is not affine and cannot be used directly as an LMI in a maxdet-problem. Hence, another matrix that is both symmetric and affine is proposed in the following.

Assume that the direction of the $x$-axis is to be restricted. Similarly to Equation (17), the requirement that the body frame $x$-axis is to point in the direction of the inertial frame $x$-axis is given by

$$
P_{2}=\left[\begin{array}{ccc}
\xi & 0 & q_{2} \\
0 & \xi & q_{3} \\
q_{2} & q_{3} & \xi
\end{array}\right] \succeq 0
$$

where $\xi=\sin \frac{\beta_{l i m}}{2}$.

Also note that the results presented are not restricted to the global reference frame $\mathcal{F}_{I}$. Assume that the direction of the body frame $x$-axis is to point in an arbitrary direction given by the direction of the $x$-axis of $Q_{d}=\left[\begin{array}{llll}d_{0} & d_{1} & d_{2} & d_{3}\end{array}\right]^{\top}$. In order to apply the restriction given by (27), but to the direction of the $x$-axis of $\mathcal{F}_{Q_{d}}$ and not that of $\mathcal{F}_{I}, Q$ is transformed back into the inertial frame and the test is performed on the transformed quaternion

$$
Q_{t}=Q_{d}^{*} * Q=\left[\begin{array}{c}
* \\
* \\
-d_{2} q_{0}+d_{0} q_{2}-d_{3} q_{1}+d_{1} q_{3} \\
-d_{3} q_{0}+d_{0} q_{3}-d_{1} q_{2}+d_{2} q_{1}
\end{array}\right]
$$

Note that when $Q_{t}$ is substituted into (27), $P_{2}$ is still symmetric and affine in $Q$.

\section{Pyramid}

Assume instead that one would like to restrict the allowed rotation differently around different axes. For example, if the set of allowed orientations is given by restrictions on the rotation about the $x$-axis followed by a rotation about the $y$-axis, this will result in a pyramid-shaped set of allowed directions. The following observations are important in the following.

Rotating the vector $\overline{\boldsymbol{v}}_{1}=\left[\begin{array}{lll}0 & 0 & 1\end{array}\right]^{\top}$ by $\alpha$ about the $x$-axis of the inertial frame followed by a rotation $\beta$ about the $y$-axis, also of the inertial frame, is given by

$$
\overline{\boldsymbol{v}}_{I}=\left[\begin{array}{c}
\cos \alpha \sin \beta \\
-\sin \alpha \\
\cos \alpha \cos \beta
\end{array}\right] .
$$

For a rotation $\alpha$ about the $x$-axis of the inertial frame followed by a rotation $\beta$ about the $y$-axis of the rotated coordinate system is given by

$$
\overline{\boldsymbol{v}}_{R}=\left[\begin{array}{c}
\sin \beta \\
-\sin \alpha \cos \beta \\
\cos \alpha \sin \beta
\end{array}\right] .
$$

This can also be written as a quaternion $Q$. Let the vector $\overline{\boldsymbol{v}}_{1}=\left[\begin{array}{lll}0 & 0 & 1\end{array}\right]^{\top}$, represent the $z$-axis, be rotated by $Q$ into
$\boldsymbol{v}_{2}=Q * \boldsymbol{v}_{1} * Q^{*}$. Then $\boldsymbol{v}_{2}$ is written as

$$
\boldsymbol{v}_{2}=\left[\begin{array}{c}
0 \\
2\left(q_{0} q_{2}+q_{1} q_{3}\right) \\
2\left(q_{2} q_{3}-q_{0} q_{1}\right) \\
q_{0}^{2}-q_{1}^{2}-q_{2}^{2}+q_{3}^{2}
\end{array}\right]
$$

This is a point on the unit sphere.

Proposition 3.3: Given a restriction $\alpha_{\text {lim }}$ in the orientation error about the $x$-axis of the inertial frame and $\beta_{l i m}$ in the orientation error about the $y$-axis of the rotated coordinate frame. Then the $z$-axis of frame $\mathcal{F}_{Q}$ rotated by the quaternion $Q=\left[\begin{array}{llll}q_{0} & q_{1} & q_{2} & q_{3}\end{array}\right]^{\top}$ with respect to the inertial frame $\mathcal{F}_{I}$ lies within the restrictions given by $\beta_{\text {lim }}$ if and only if

$$
P_{1}=\left[\begin{array}{ccc}
\eta & 0 & q_{1} \\
0 & \eta & q_{0} \\
q_{3} & q_{2} & \eta
\end{array}\right] \geq 0
$$

where $\eta=\sqrt{\frac{\sin \beta_{l i m}}{2}}$ and $\geq$ means positive semi-definiteness for the non-symmetric matrix $P_{1}$.

Proof: The determinant of $P_{1}$ is given by

$$
\operatorname{det}\left(P_{1}\right)=\eta\left(\eta^{2}-q_{0} q_{2}-q_{1} q_{3}\right) .
$$

Assume $\operatorname{det}\left(P_{1}\right) \geq 0$

$$
\begin{gathered}
\eta^{2}-q_{0} q_{2}-q_{1} q_{3} \geq 0 \\
\eta^{2} \geq q_{0} q_{2}+q_{1} q_{3}
\end{gathered}
$$

As $\beta_{\text {lim }} \geq 0$, comparing Equations (30) and (31) gives

$$
\sin \beta_{\text {lim }} \geq 2\left|q_{0} q_{2}+q_{1} q_{3}\right| \text {. }
$$

Let

$$
\beta^{\prime}=\arcsin \left(2\left(q_{0} q_{2}+q_{1} q_{3}\right)\right),
$$

and the initial requirement is obtained by

$$
-\beta_{\text {lim }} \leq \beta^{\prime} \leq \beta_{\text {lim }}
$$

where $\beta^{\prime}$ is the angle between the new $z$-axis and the $y z$ plane.

Proposition 3.4: Given a restriction $\alpha_{l i m}$ in the orientation error about the $x$-axis and $\beta_{\text {lim }}$ in the orientation error about the $y$-axis, both in the inertial frame. Then the $z$-axis of frame $\mathcal{F}_{Q}$ rotated by the quaternion $Q=\left[\begin{array}{llll}q_{0} & q_{1} & q_{2} & q_{3}\end{array}\right]^{\top}$ with respect to the inertial frame $\mathcal{F}_{I}$ lies within the restrictions given by $\alpha_{\text {lim }}$ if and only if

$$
P_{2}=\left[\begin{array}{ccc}
\xi & q_{2} q_{3} & 0 \\
q_{2} q_{3} & \xi & q_{0} q_{1} \\
2 \xi & q_{0} q_{1} & \xi
\end{array}\right] \geq 0
$$

where $\xi=\frac{\sin \alpha_{l i m}}{2}$.

Proof: The determinant of $P_{2}$ is given by

$$
\operatorname{det}\left(P_{2}\right)=\xi\left(\xi^{2}-\left(q_{0} q_{1}\right)^{2}-\left(q_{2} q_{3}\right)^{2}+2 q_{0} q_{1} q_{2} q_{3}\right) .
$$

Assume $\operatorname{det}\left(P_{1}\right) \geq 0$

$$
\begin{gathered}
\xi^{2}-\left(q_{0} q_{1}\right)^{2}-\left(q_{2} q_{3}\right)^{2}+2 q_{0} q_{1} q_{2} q_{3} \geq 0 \\
\xi^{2} \geq\left(q_{0} q_{1}\right)^{2}+\left(q_{2} q_{3}\right)^{2}-2 q_{0} q_{1} q_{2} q_{3} \\
\xi^{2} \geq\left(q_{0} q_{1}-q_{2} q_{3}\right)^{2} \\
|\xi| \geq\left|q_{0} q_{1}-q_{2} q_{3}\right|
\end{gathered}
$$


As $\alpha_{l i m} \geq 0$, comparing Equations (31) and (29) gives

$$
\sin \alpha_{l i m} \geq 2\left|q_{0} q_{1}-q_{2} q_{3}\right| .
$$

Let

$$
\alpha=\arcsin \left(2\left(q_{0} q_{1}-q_{2} q_{3}\right)\right),
$$

and the initial requirement is obtained by

$$
-\alpha_{l i m} \leq \alpha \leq \alpha_{l i m} .
$$

Note that in Proposition 3.3 the second rotation is with respect to the rotated coordinate frame and restricts only the rotations about the $y$-axis while in Proposition 3.4 the second rotation is with respect to the rotated coordinate frame and restricts the allowed rotations about the $x$-axis only. This simplifies the computations substantially. For small $\alpha$ and $\beta$ this is a good approximation. The next step is to put the two restrictions together to one block-diagonal matrix. This shows how two restrictions on the orientation can be put together and represented as one constraint.

Note that the matrices given in Propositions 3.3 and 3.4 are not symmetric and that $P_{2}$ in (38) is not affine. Hence, the constraints cannot be represented as LMIs. They can, however, be represented as barrier functions given as the negative logarithm of the determinant. This is discussed shortly in Section V.

Example 1: Given a restriction $\alpha_{\text {lim }}$ in the orientation error about the $x$-axis and $\beta_{\text {lim }}$ in the orientation error about the $y$ axis. Then the $z$-axis of frame $\mathcal{F}_{Q}$ rotated by the quaternion $Q=\left[\begin{array}{llll}q_{0} & q_{1} & q_{2} & q_{3}\end{array}\right]^{\top}$ with respect to the inertial frame $\mathcal{F}_{Q}$ lies within the restrictions given by $\alpha_{l i m}$ and $\beta_{l i m}$ if and only if

$$
P=\left[\begin{array}{cc}
P_{1} & 0 \\
0 & P_{2}
\end{array}\right] \geq 0
$$

where $P_{1}$ and $P_{2}$ are given as in Equations (32) and (38).

Alternatively, if one would like to restrict the orientation about the $x$-axis followed by the orientation about the new $y$-axis, this can be achieved by substituting

$$
\eta=\sqrt{\frac{\sin \alpha_{\text {lim }} \cos \beta}{2}}
$$

where $\beta=\arcsin 2\left(q_{0} q_{2}+q_{1} q_{3}\right)$ for $\eta$ into Equation (32) which will give the exact solution.

\section{ApPLiCATIONS}

In this section it is shown how the results from the previous section can be used as LMIs in an optimisation problem.

\section{A. Analytic Centering}

The problem

$$
\begin{array}{ll}
\text { minimise } & \phi(x)=\log \operatorname{det} G(x)^{-1} \\
\text { subject to } & G(x) \succ 0
\end{array}
$$

where

$$
G(x)=G_{0}+x_{1} G_{1}+x_{2} G_{2}+\cdots+x_{m} G_{m},
$$

is known as the analytic centering problem. If the feasible set $\boldsymbol{X}=\{x \mid G(x) \succ 0\}$ is non-empty and bounded, then the matrices $G_{i}, i=1, \ldots, m$ are linearly independent and the objective function is strictly convex on $\boldsymbol{X}$ [7]. In this case, it can be guaranteed that the optimality condition $\nabla \phi\left(x^{*}\right)=0$, for an optimal solution $x^{*}$, can be reached.

In our case, for the specification of the $z$-axis:

$$
\begin{array}{ll}
\text { minimise } & \phi(x)=\log \operatorname{det} P(x)^{-1} \\
\text { subject to } & P(x) \succ 0
\end{array}
$$

where $P$ is given by Equation (17) and can be written as

$$
P(x)=P_{0}+x_{1} P_{1}+x_{2} P_{2}+x_{3} P_{3}+x_{4} P_{4},
$$

where

$$
\begin{gathered}
x_{1}=q_{0}, \quad x_{2}=q_{1}, \quad x_{3}=q_{2}, \quad x_{4}=q_{3}, \\
P_{0}=\left[\begin{array}{lll}
\eta & 0 & 0 \\
0 & \eta & 0 \\
0 & 0 & \eta
\end{array}\right], \quad P_{1}=P_{4}=\left[\begin{array}{lll}
0 & 0 & 0 \\
0 & 0 & 0 \\
0 & 0 & 0
\end{array}\right], \\
P_{2}=\left[\begin{array}{lll}
0 & 0 & 1 \\
0 & 0 & 0 \\
1 & 0 & 0
\end{array}\right], \quad P_{3}=\left[\begin{array}{lll}
0 & 0 & 0 \\
0 & 0 & 1 \\
0 & 1 & 0
\end{array}\right] .
\end{gathered}
$$

$q_{0}$ and $q_{3}$ do not affect the solution and can be eliminated from the equations.

The optimal solution to this problem is given by the set

$$
Q_{\text {opt }}=\left\{Q \mid q_{1}=q_{2}=0\right\}
$$

or similarly by all quaternions on the form

$$
Q_{o p t}=\left[\begin{array}{llll}
\cos \frac{\psi}{2} & 0 & 0 & \sin \frac{\psi}{2}
\end{array}\right]^{\top} .
$$

The solution to this problem is trivially given by all the orientations that make the body frame $z$-axis point in the direction of the inertial frame $z$-axis. We now look into the case when an additional constraint is added. Two cases are considered; (a) two constraints are represented by the positive definiteness of two matrices $P_{1}$ and $P_{2}$ and the determinant of the block diagonal matrix $P=\operatorname{Blockdiag}\left(P_{1}, P_{2}\right)$ is minimised; and (b) $P_{1}$ is minimised and $P_{2}$ is a constraint.

\section{B. Blockdiagonal G-matrix}

To combine the restrictions of the $x$ - and $z$-axes, substitute (28) into (27), denote the resulting matrix $F$, and write it on the form of (49) so that

$$
\begin{gathered}
F(x)=F_{0}+x_{1} F_{1}+x_{2} F_{2}+x_{3} F_{3}+x_{4} F_{4}, \\
x_{1}=q_{0}, \quad x_{2}=q_{1}, \quad x_{3}=q_{2}, \quad x_{4}=q_{3}, \\
F_{0}=\left[\begin{array}{lll}
\xi & 0 & 0 \\
0 & \xi & 0 \\
0 & 0 & \xi
\end{array}\right], \quad F_{1}=\left[\begin{array}{ccc}
0 & 0 & -d_{2} \\
0 & 0 & -d_{3} \\
-d_{2} & -d_{3} & 0
\end{array}\right], \\
F_{2}=\left[\begin{array}{ccc}
0 & 0 & -d_{3} \\
0 & 0 & d_{2} \\
-d_{3} & d_{2} & 0
\end{array}\right], \quad F_{3}=\left[\begin{array}{ccc}
0 & 0 & d_{0} \\
0 & 0 & -d_{1} \\
d_{0} & -d_{1} & 0
\end{array}\right], \\
F_{4}=\left[\begin{array}{ccc}
0 & 0 & d_{1} \\
0 & 0 & d_{0} \\
d_{1} & d_{0} & 0
\end{array}\right] .
\end{gathered}
$$


The problem can now be formulated as follows:

$$
\begin{array}{ll}
\text { minimise } & \phi(x)=\log \operatorname{det}\left[\begin{array}{cc}
P(x) & 0 \\
0 & F(x)
\end{array}\right]^{-1} \\
\text { subject to } & {\left[\begin{array}{cc}
P(x) & 0 \\
0 & F(x)
\end{array}\right] \succ 0}
\end{array}
$$

for which the solution is the orientation which minimises the error both of the $x$-axis and the $z$-axis with a "metric" that increases exponentially with the shortest angular distance from the desired directions of the $x$ - and $z$-axes.

\section{LMI Constraint}

Alternatively the determinant of $P$ can be minimised under the constraint $F(x) \succeq 0$.

$$
\begin{array}{ll}
\text { minimise } & \phi(x)=\log \operatorname{det} P(x)^{-1} \\
\text { subject to } & P(x) \succ 0 \\
& F(x) \succeq 0 .
\end{array}
$$

Hence, the optimal solution of the direction of the $z$-axis is found and the direction of the $x$-axis is within the restrictions. If the $x$-axis is close or far from the desired direction does not affect the solution.

\section{Normalisation}

The optimisation algorithms described optimise freely over all quaternions, and it is thus not guaranteed, nor likely, that the resulting quaternion is of unit length. One simple, though not very mathematically sound solution is to optimise freely over all quaternions and then normalise the result afterwards. Another option is to add the constraint $|Q|=1$ in the optimisation algorithm which guarantees that the search space is only the set of quaternions of unit length. For the restriction given by Equation (17), it is for example sufficient to add the restriction

$$
q_{1}^{2}+q_{2}^{2} \leq 1
$$

\section{Future WORK}

The examples shown in this paper are all very basic. They do, however, show how the formulation allows the programmer to include the constraints on the orientation error in optimisation problems in the form of linear matrix inequalities. Constraints in the form of LMIs are convenient in the sense that they are easily combined with other LMIs into one "big" LMI. This is exploited in for example [9].

Consider the LMI

$$
\operatorname{Blockdiag}\left(P_{1}(x), P_{2}(x), \ldots, P_{k}(x)\right) \succeq 0 .
$$

Let $P_{1}\left(x_{1}\right)$ be the constraint on the orientation error. Then, $P_{2}(x) \ldots P_{k}(x)$ may impose other constraints on the optimisation problem. These may be related to the orientation, as in (55). The true advantage of representing the constraints as LMIs, however, only come apparent when the constraints on the orientation is combined with other constraints such as joint torque or the quality of the job performed. This is left as future research topics.

In this paper only LMIs have been considered, but the restrictions on the orientation could just as well have been formulated as for example barrier functions. Given the restrictions in Proposition 3.1, this can be formulated as a the barrier for the cone constraint

$$
\phi=-\log \left(\eta^{2}-q_{1}^{2}-q_{2}^{2}\right)
$$

which is then included in the barrier subproblem

$$
\begin{array}{ll}
\text { minimise } & F(x)+\phi(x) \\
\text { subject to } & P(x)=0
\end{array}
$$

where $F(x)$ is the objective function that we want to minimise and $P(x)$ is some additional constraint.

\section{CONCLUSIONS}

This paper casts constraints on the orientation error into linear matrix inequalities. For many practically important examples, it has been shown that the continuous set of orientations that satisfy a given constraint can be represented by a positive definiteness constraint of a certain matrix where the four real quaternion-quantities $q_{0}, q_{1}, q_{2}$ and $q_{3}$ are the entries. Some simple examples of how to include the LMIs into the maxdet-problem are given. The LMIs can be included in optimisation algorithms to find the optimal orientation of some devise, such as a satellite or the end effector of a robotic manipulator, subject to some optimisation criteria.

\section{ACKNOWLEDGMENT}

The authors wish to acknowledge the support of the Norwegian Research Council and the TAIL IO project for their continued funding and support for this research. The TAIL IO project is an international cooperative research project led by StatoilHydro and an R\&D consortium consisting of $A B B$, IBM, Aker Kvaerner and SKF. During the work with this paper the first author was with the Hong Kong University of Science and Technology.

\section{REFERENCES}

[1] V. Potkonjak, G. Dordevic, D. Kostic and M. Rasic, Dynamics of anthropomorphic painting robot: Quality analysis and cost reduction Robotics and Autonomous Systems, Vol. 32, No 1, 2000.

[2] P. J. From and J. T. Gravdahl, General solutions to Kinematic and Functional Redundancy Proc. 46th IEEE Conf. on Decision and Control, 2007.

[3] M. Buss, H. Hashimoto and J. B. Moore, Dextrous Hand Grasping Force Optimization IEEE Trans. on robotics and automation Vol. 12 no. 3, 1996.

[4] B. Alpern, L. Carter and M. Grayson and C. Pelkie, Orientation Maps: Techniques for Visualizing Rotations (A Consumers Guide) IEEE Conference on Visualization, 1993.

[5] J. B. Kuipers, Quaternions and Rotation Sequences Princeton University Press, 2002.

[6] J. M. Ahuactzin and K. K. Gupka, The Kinematic Roadmap: A Motion Planning Based Global Approach for Inverse Kinematics of Redundant Robots IEEE Trans. on Robotics and Automation, 1999.

[7] L. Vandenberghe, $\mathrm{S}$ Boyd and S. P. Wu, Determinant maximization with linear matrix inequality constraints http://stanford.edu/ boyd/papers/maxdet.html, 1996.

[8] P. J. From and J. T. Gravdahl, Representing Attitudes as Sets of Frames Proc. American Control Conference, 2007.

[9] L. Han, J. Trinkle and Z. Li, Grasp analysis as linear matrix inequalitiy problem IEEE Transactions on Robotics and Automation Vol. 16, no 6, 2000. 